\title{
Die disziplinären Grundlagen der Wissenschaft
}

Alfons Bora 



\title{
Die disziplinären Grundlagen der Wissenschaft
}

\author{
Alfons Bora \\ Institut für Wissenschafts- und Technikforschung (IWT), Universität Bielefeld
}

\section{Keywords}

Wissenschaft, Disziplinen, Interdisziplinarität, Transdisziplinarität, Technology Assessment

\begin{abstract}
Die Begriffe der Inter- und Transdisziplinarität prägen die Beschreibung der modernen Wissenschaft. Vor dem Hintergrund eines kurzen historischen Rückblicks wird mit Blick auf die gegenwärtige Wissenschaft die These entwickelt, dass wir sowohl mit dem Begriff der Inter- als auch dem der Transdisziplinarität die Einheit einer Differenz beschreiben, im einen Fall einer innerwissenschaftlichen Differenz und im anderen der zwischen Wissenschaft und ihrer Umwelt. Daher stellt sich die Frage, worin diese Einheit besteht. Sie besteht, so wird argumentiert, aus denselben Elementen der Wissenschaftlichkeit, die auch für alle Disziplinen konstitutiv sind. Die Rolle disziplinärer und disziplinförmiger Strukturen wird daher als zentral für die moderne Wissenschaft verstanden. Diese Sichtweise wird entlang dreier theoretisch-begrifflicher Argumentationslinien plausibilisiert, die sich mit den Stichworten Interdisziplinarität, Transdisziplinarität und Transwissenschaft kennzeichnen lassen. Als empirischer Fall und Prüfstein dient dabei das Beispiel der TA.
\end{abstract}




\section{Inhalt}

1 Neue Formen der Wissensproduktion? ...................................................................... 3

2 Die disziplinäre Einheit der modernen Wissenschaft .................................................... 5

3 Inter- und Transdisziplinarität: Eine neue Einheit der Wissenschaft?.................................... 9

4 Die wissenschaftlichen Grundlagen von Transwissenschaft ............................................... 15

5 Zusammenfassung: Disziplinbasierung und Disziplinförmigkeit ........................................ 18

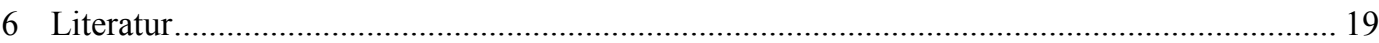

Der Text basiert auf einem Vortrag im Rahmen der Konferenz „,TA'07 - Technikfolgenabschätzung zwischen Inter- und Transdisziplinarität" am 4. Juni 2007 an der Österreichischen Akademie der Wissenschaften in Wien.

\section{IMPRESSUM}

\section{Medieninhaber:}

Österreichische Akademie der Wissenschaften

Juristische Person öffentlichen Rechts (BGBI 569/|921 idF BGBI I | 30/2003)

Dr. Ignaz Seipel-Platz 2, A-1010 Wien

\section{Herausgeber:}

Institut für Technikfolgen-Abschätzung (ITA)

Strohgasse 45/5, A- 1030 Wien

http://www.oeaw.ac.at/ita

Die ITA-manu:scripts erscheinen unregelmäßig und dienen der Veröffentlichung

von Arbeitspapieren und Vorträgen von Institutsangehörigen und Gästen.

Die manu:scripts werden ausschließlich über das Internetportal „epub.oeaw“

der Öffentlichkeit zur Verfügung gestellt:

http://epub.oeaw.ac.at/ita/ita-manuscript

ITA-manuscript Nr.: ITA-07-08 (November/2007)

ISSN-online: I 8| 8-6556

http://epub.oeaw.ac.at/ita/ita-manuscript/ita_07_08.pdf

c 2007 ITA - Alle Rechte vorbehalten 


\section{Neue Formen der Wissensproduktion?}

Inter- und Transdisziplinarität prägen nicht nur die Selbstbeschreibung der Technikfolgenabschätzung und -bewertung (im folgenden TA). Sie prägen vielfach auch die Beschreibung der modernen Wissenschaft überhaupt. Das Lob der disziplinären Entdifferenzierung gehört wissenschaftssoziologisch inzwischen fast zum guten Ton. Neue Formen der Wissensproduktion werden beobachtet (Gibbons et al. 1994, Nowotny et al. 2001) und als Indizien für eine post-normale Wissenschaft (Funtowicz/Ravetz 1993, Ravetz 1999, 2004) gedeutet. Die aus dem neunzehnten Jahrhundert überlieferte Struktur der Disziplinen gilt dementsprechend auch in der Wissenschaftsorganisation als überholt. Disziplinen gelten als Ursache verengter, einseitiger, nicht problemangemessener Sichtweisen in der Wissenschaft. Inter- und Transdisziplinarität werden als angemessene Reaktionen auf derartige Verkrustungsphänomene interpretiert (vgl. bspw. die Einleitung von Grunwald und Schmidt 2005). Aber nicht nur die Selbstbeschreibung der Wissenschaft stellt sich in der skizzierten Weise auf eine Welt jenseits disziplinärer Differenzierungen ein. Auch die Organisationen der Wissenschaft allgemein und des Hochschulsystems im Besonderen scheinen Diagnosen eines postdisziplinären Zeitalters zu bestätigen. So lässt sich beispielsweise zeigen (Brint 2005, 46), wie eine große Zahl ehemals eher mittelmäßiger Universitäten in den USA versucht, mittels neuer interund transdisziplinärer Forschungs- und Lehrgebiete und der Gründung entsprechender „Zentren“ den Sprung auf die vorderen Plätze der Universitäts-Rankings zu schaffen, bisweilen sogar mit beachtlichem Erfolg.

Diese Entwicklungen geben Anlass, über die Funktion der Disziplinen nachzudenken und zu fragen, welche Rolle sie in emergenten Feldern der Wissenschaft noch spielen können, zu denen unter anderem auch die TA zählt. Denn gerade solche vergleichsweise neuen Felder werden immer wieder als Beleg für die diagnostizierten Trends der Wissenschaftsentwicklung angeführt. Im Unterschied zu solchen Trenddiagnosen wird in diesem Beitrag die Sichtweise vertreten, dass Wissenschaft in konstitutiver Weise auf Disziplinen oder deren funktionale Äquivalente angewiesen ist, welche sich im Kern zumindest durch Disziplinförmigkeit - ein materiales Feld, typische Fragestellungen und eine integrative Theoriebildung - auszeichnen. Diese Ansicht ist - wenngleich nicht unbedingt populär - in der Wissenschaftsforschung verschiedentlich vertreten worden, etwa von Abbott (2001). Im Gegensatz etwa zu Abbotts Position, der mit Strukturgesetzlichkeiten der Wissenschaftsorganisation argumentiert, wird im vorliegenden Beitrag ein eher funktionalistisches Argument im Hinblick auf die Erzeugung der Einheit von Wissenschaft in Anschlag gebracht. Um dieses Argument einigermaßen plausibel vertreten zu können, wird zunächst die Semantik der Inter- und Transdisziplinarität selbst genauer zu betrachten sein. In welchem Verhältnis stehen Interund Transdisziplinarität zueinander und zur klassischen Form der Disziplinen? Was bezeichnen die Deutungsmuster jeweils? Wogegen grenzen sie sich ab? Worin kann ihre spezifische Leistungsfähigkeit für die Wissenschaft bestehen? Sodann kann gefragt werden, in welchen Formen Inter- und Transdisziplinarität möglich sind und welche Bedeutung sie für die moderne Wissenschaft haben können.

Vor dem Hintergrund eines kurzen historischen Rückblicks wird im folgenden mit Blick auf die gegenwärtige Wissenschaft die These entwickelt und in aller Knappheit begründet, dass wir sowohl im Falle der Inter- wie auch der Transdisziplinarität die Kommunikation der Einheit einer Differenz beobachten, dessen also, was gewissermaßen die Klammer ausmacht, die zwei Seiten einer Unterscheidung zusammenhält. Im einen Fall geht es dabei um innerwissenschaftliche Differenz und im anderen um die Differenz zwischen Wissenschaft und ihrer sozialen Umwelt. Wenn eine solche voraussetzungsreiche und paradoxieverdächtige Kommunikationsfigur ein gelingendes Deutungsmuster wissenschaftlichen Handelns abgeben soll - und dass dafür Bedarf besteht, soll gar nicht bestritten werden - erfordert sie ein hohes Maß an wissenschaftlicher Selbstreflexion im 
Inneren und eine spezifische Form der Professionalisierung nach außen. Technology Assessment im Speziellen, aber auch die Wissenschaft im Allgemeinen hat deshalb Anlass, sich selbstreflexiv der disziplinären Grundlagen ihres Handelns zu versichern und ein schlüssiges Modell inter- und transdisziplinären Operierens zu entwickeln, bevor sie beide als normative Ziele ihres Handelns wählt.

Im Gegensatz zu den eingangs erwähnten Zeitdiagnosen wird, wie bereits erwähnt, im folgenden die Rolle disziplinärer und disziplinförmiger Strukturen als zentral für die moderne Wissenschaft verstanden. Diese Sichtweise wird entlang dreier theoretisch-begrifflicher Argumentationslinien plausibilisiert, die sich mit den Stichworten Interdisziplinarität, Transdisziplinarität und Transwissenschaft kennzeichnen lassen. Als empirischer Fall und Prüfstein dient dabei das Beispiel der TA.

Interdisziplinarität, so die erste Argumentationslinie, beschreibt entweder die - wie immer en detail beschaffene - Kooperation zwischen autonomen Disziplinen oder die Bildung einer neuen disziplinförmigen Ebene wissenschaftlichen Handelns, die in ihrer Disziplinförmigkeit die Einheit der Differenz bestehender Disziplinen abbildet. Beide Varianten ruhen, wenn auch in unterschiedlichem Sinne, auf disziplinförmigen Grundlagen auf.

Die zweite Variante einer neuen disziplinförmigen Ebene wissenschaftlichen Handelns nenne ich unter Rekurs auf Jantsch und Mittelstraß, aber doch auch über sie hinausgehend, Transdisziplinarität. Dies ist die eben erwähnte zweite Argumentationslinie.

Transdisziplinarität im solchermaßen spezifizierten Sinne ist weiterhin in einer dritten Argumentationslinie zu unterscheiden von allen Formen der Transwissenschaft, also des Operierens außerhalb wissenschaftlicher Sinnhorizonte. Diese Formen - die oft ebenfalls mit dem Begriff Transdisziplinarität belegt werden - stehen im Zentrum des Interesses von Theorien der post normal science oder des Mode 2. Sie beruhen entgegen den Thesen, die dort vertreten werden, ebenfalls auf wissenschaftlichen Grundlagen, soweit sie darauf abzielen, wissenschaftsrelevante Erkenntnisse zu produzieren.

Wenn eingangs gesagt wurde, dass das Interesse an Inter- und Transdisziplinarität unter anderem durch das Aufkommen hybrider Forschungsfelder wie beispielsweise der TA erklärt wird, so soll abschließend die empirische Vermutung geäußert und plausibilisiert werden, dass Technology Assessment im Schnittfeld der drei genannten Formen liegt, also der Kooperation autonomer Disziplinen, der Ausbildung einer transdisziplinären Einheit und der Transwissenschaft. Für das Funktionieren aller drei Formen sind jedoch, wie aus den drei eben genannten Argumentationslinien folgt, disziplinäre oder disziplinförmige Mechanismen unabdingbar. Auch im Falle der TA wird also davon auszugehen sein, dass wissenschaftliches Handeln in fundamentaler Weise auf Disziplinen angewiesen bleibt und allfällige Neuorientierungen wissenschaftlichen Handelns sich im Schatten der Diziplinen vollziehen.

Die Darstellung orientiert sich in ihrem Aufbau an den genannten drei Argumentationslinien. Im ersten Schritt wird an die Ausdifferenzierung der Disziplinen und ihre Funktion erinnert (Abschn. 2). Im zweiten Schritt wird gefragt, auf welches Problem die Entstehung von Inter- bzw. Transdisziplinarität reagiert und welche Formen diese annehmen (Abschn. 3). Der dritte Schritt betrifft das Phänomen der Transwissenschaft, das die Wissenschaftssoziologie derzeit so beunruhigt und das für TA eine erhebliche praktische Bedeutung hat (Abschn. 4.). Das Verständnis von Transwissenschaft wird, so die Vermutung, mit Hilfe der hier vorgeschlagenen Begrifflichkeiten erleichtert. Es ermöglicht abschließend eine knappe Diagnose des Zustandes von TA vor dem Hintergrund der vorgenommenen Begriffsbestimmungen. Diese Einordnung ist als Versuch zu verstehen, die wissenschaftssoziologische Debatte über Inter- und Transdisziplinarität, die in gewisser Weise festgefahren ist, mit neuem Problempotential zu versorgen. Keinesfalls kann es sich um ein abschließendes Urteil handeln, das sich in derart komplexen Fragen ohnehin verbieten dürfte, sondern bestenfalls um begrifflich sortierte und theoretisch einigermaßen plausible Überlegungen zu Funktion und Form von Inter- und Transdisziplinarität. 


\section{Die disziplinäre Einheit der modernen Wissenschaft}

Bevor nach der Entstehungsgeschichte von Inter- und Transdisziplinaritätsdebatten gefragt werden kann, empfiehlt es sich, Entstehungsgeschichte, Struktur und Funktion der wissenschaftlichen Disziplinen noch einmal kurz in Erinnerung zu rufen. Nur vor diesem Hintergrund ist es möglich, den Problemkontext zu rekonstruieren, auf den die disziplinüberschreitenden Phänomene eine Reaktion darstellen könnten. Wenn man sich also fragt, was eigentlich unter Disziplinen zu verstehen ist und welche Probleme diese Form der Binnendifferenzierung im Funktionssystem der Wissenschaft zu bearbeiten hilft, stößt im Ergebnis auf einen Befund, der sich wie folgt zusammenfassend skizzieren lässt:

Disziplinen symbolisieren vor dem Hintergrund einer hohen internen Komplexität des Wissenschaftssystems jeweils - also jede Disziplin für sich - die Einheit der Wissenschaft, die als solche, nämlich als inhaltliche Einheit der Wissenschaft insgesamt nicht mehr zur Verfügung steht, jedoch für das Funktionieren des Wissenschaftssystems eine wichtige Voraussetzung darstellt. Disziplinen sind also Vorkehrungen, die es ermöglichen, angesichts einer Vielzahl von Forschungsfeldern und Fächern im je konkreten Fall die Grenze zwischen Wissenschaft und ihrer sozialen Umwelt zu markieren. Sie bilden sich historisch während des 18. und 19. Jahrhunderts im Zuge der Ausdifferenzierung des Wissenschaftssystems, das heißt seiner Abgrenzung nach außen. Sie bilden diese Abgrenzung noch einmal $\mathrm{ab}$ und ermöglichen sie unter Bedingungen fortschreitender Komplexität dadurch überhaupt erst. Sie entstehen damit aus den internen Erfordernissen (der Selbstrepräsentation bzw. Selbstreflexion) eines sich ausdifferenzierenden Funktionssystems unter der Bedingung von Größenwachstum.

Diese theoretische Perspektive auf die Entstehung der Disziplinen soll im Folgenden in aller Kürze an Hand eines Rückblicks auf die Geschichte der Disziplinenbildung zwischen dem 17. und 19. Jahrhundert erläutert werden. Bei der Abgrenzung des Gegenstandes schließe ich mich dabei in definitorischer Hinsicht zunächst einem Vorschlag von Heinz Heckhausen an $(1987,131)$ und bestimme Disziplin als Form der Binnendifferenzierung des Wissenschaftssystems, die sich durch ein materiales Feld (Gegenstandsbereich), einen spezifischen Gegenstandsaspekt (typische Fragestellungen) sowie durch integrative Theoriebildung (paradigmenförmige Theorie oder Theorien) auszeichnet. ${ }^{1}$ Außerdem umfassen Disziplinen in sozialstruktureller Hinsicht all jene organisatorischen Aspekte, die sich um derartige „kulturelle Kohäsionsachsen“ herum bilden. Abbott $(2001,140)$ bezeichnet dieses Ensemble aus sozialstrukturellen (Organisationen, und deren Personal, Vereinigungen und Verbände) und kulturellen (Themen, Fragen, Theorien) Eigenschaften als ,settlement“. Mit dieser Abgrenzung sind die wesentlichen Merkmale aller Phänomene erfasst, die im Allgemeinen und unstreitig als Disziplinen bezeichnet werden.

Dabei ist der Begriff „Disziplin“ selbst wesentlich älter als das moderne Phänomen der Disziplin. Die Fähigkeit des modernen Wissenschaftssystems, über interne Differenzierung seine eigene Ordnung zu bilden, ist Resultat eines evolutionären Prozesses, in dem sich erst spät das uns heute (noch) vertraute Gefüge wissenschaftlicher Disziplinen bildet. Der Begriff „Disziplin“ hingegen bezeichnet seit der Antike ganz allgemein das in lehrbare Form gebrachte Wissen (Stichweh 1984, 9). Das Verständnis der historisch gewachsenen Funktion von Disziplinarität hängt deshalb in erster Linie von der Funktion der Lehre ab. Für wen und mit welchem Ziel wurde gelehrt? In der spätmittelal-

1 Der Begriff der Binnendifferenzierung ist theoretisch noch unscharf. Hinweise auf aussichtsreich erscheinende konzeptionelle Varianten sind andernorts unter den Stichworten „Diskurs“ und „soziale Positionierung“ gegeben worden (Bora 1999, 2000, 2005; Hausendorf/Bora 2006). Sie bedürften weiterer Ausarbeitung. 
terlichen Universität fällt die Antwort unterschiedlich aus, je nachdem welche Fakultät man im Blick hat. Die drei höheren Fakultäten - Theologie, Jurisprudenz und Medizin - lehrten immer schon mit einem doppelten Ziel, nämlich einerseits zur Produktion wissenschaftlichen Nachwuchses und andererseits zur Produktion außerwissenschaftlich brauchbaren Wissens. Diese in der Fakultätsgeschichte angelegte Doppeldeutigkeit der Ziele führte sehr viel später dazu, dass sich in den genannten Fakultäten der Begriff des Gelehrten in den nach außen gerichteten Professionellen einerseits und den nach innen orientierten Fachwissenschaftler andererseits spaltet. Auf diese Entwicklung wird später unter dem Gesichtspunkt der Transwissenschaft zurückzukommen sein.

Die Außenwirkung der höheren Fakultäten mündet noch ungebrochen in das im 17. Jahrhundert zur Blüte kommende Modell der Universität als Staatsdienerschule, in welcher nützliche - und das heißt spätestens seit der Einführung der Kameralwissenschaft und der Ökonomie auch empirische - Wissenschaft betrieben und das Gelehrte in den nunmehr von den Territorialstaaten beherrschten Universitäten in Staatsexamina abgeprüft wurde.

Bis in diese Phase hinein kann man die innere Ordnung des Wissenschaftssystems mit Stichweh (1984, 18 ff.) als Hierarchie von Wissensformen charakterisieren, die durch äußere ordnende Zugriffe zustande gekommen war und sich im System der Fakultäten abbildete. Dabei sind die Ordnung des Wissens und dessen wissenschaftsinterne Organisation durch die Annahme einer universellen, kosmischen Ordnung der Dinge selbst vorgegeben, der sich das Gefüge der Wissenschaften letztlich nur anpasst.

Diese überkommene Ordnung wird in der Folgezeit mit der funktionalen Ausdifferenzierung des Wissenschaftssystems hinfällig, das sich zunehmend an inneren Kriterien orientiert und sich von externen Ordnungsleistungen unabhängig macht. Dabei spielt vor allem die Aufwertung der philosophischen Fakultät eine wichtige Rolle. Diese Aufwertung geschieht in zweierlei Hinsicht, nämlich (a) einerseits durch die Autonomisierung der Erfahrungswissenschaften und (b) andererseits durch das Versprechen der Philosophie, die Einheit der Wissenschaft angesichts wegfallender äußerer Ordnungsprinzipien zu sichern.

\section{(a) Autonomisierung der Erfahrungswissenschaften}

In der mittelalterlichen Universität hatte die Artisten-Fakultät mit ihren septem artes liberales, also Rhetorik, Grammatik und Dialektik im Trivium sowie Geometrie, Astronomie, Arithmetik und Musik im Quadrivium im Wesentlichen die wissenschaftliche Propädeutik und die Grundlagen wissenschaftlichen Handelns zum Gegenstand. Seit dem 17. Jahrhundert entwickeln sich innerhalb dieser Fakultät bekanntlich die neuen Erfahrungswissenschaften (Münte 2004). Das überkommene Muster der Fakultäten wird dadurch nun nicht nur um eine neue Komponente qualitativ erweitert. Es erfährt vielmehr auch einen quantitativen Zuwachs, der im 18. und 19. Jahrhundert enorme Ausmaße annimmt. Dieses Größenwachstum schafft „Überlastungen und Integrationsprobleme im Kommunikationssystem der Wissenschaft (...), die durch Innendifferenzierung in eine lösbare Form überführt werden“" (Stichweh 1984, 53). Diese Form wird in Gestalt von Disziplinen im Wissenschaftssystem selbst erzeugt. Den Disziplinen fällt damit die Aufgabe zu, für das Wissenschaftssystem ein hinreichendes Ausmaß an interner strukturierer Komplexität in sozialstruktureller wie kultureller Hinsicht zu garantieren. Ashby und Ross (1956, 206 ff.) haben in der allgemeinen Kybernetik dieses Niveau ausreichender innerer Komplexität mit dem Begriff „requisite variety“ gekennzeichnet. Diese ist nötig, da für jedes System die System-Umwelt-Relation ein Komplexitätsgefälle darstellt, dessen Verminderung einzig durch den Aufbau von Eigenkomplexität im System gelingt. Solche Systemkomplexität wird erzeugt durch die Einschränkung von Verknüpfungsmöglichkeiten zwischen den Systemelementen. Sie ist, wenn wir derartige Selektionseinschränkungen mit dem Begriff der Struktur belegen, also stets strukturierte Komplexität. Abbotts ,,settlements“ sorgen demnach aus einer etwas abstrakteren Perspektive für die Bereitstellung von „,requisite variety“. 
Die hierarchische Ordnung der Fakultäten macht damit im Ergebnis einer eher „,horizontalen“ Differenzierung Platz, in welcher zahlreiche Disziplinen nebeneinander existieren, die alle lediglich die Orientierung an Wissenschaftlichkeit - am „Gesetz der Vernunft“ - gemeinsam haben, sich im Übrigen aber zunächst an Gegenständen und später an Problemstellungen je eigenen Zuschnitts orientieren. Auf Grund ihrer Vorgeschichte sind die Disziplinen in dieser Entstehungsphase, wie Luhmann $(1990,449)$ sagt, einerseits noch nach außerwissenschaftlichen Relevanzen wie beispielsweise „Verwertbarkeit am Arbeitsmarkt und in beruflichen Positionen“ bestimmt und insofern noch nicht rein wissenschaftlich legitimiert. Andererseits stellen sie aber auch schon eine frühe Form der inneren Differenzierung des Wissenschaftssystems bereit, von der dieses bei seiner weiteren Ausdifferenzierung gegenüber anderen Funktionssystemen Gebrauch machen kann. Denn diese Ausdifferenzierung schreitet umso weiter voran, je mehr die Disziplinen sich nach eigenen Problemstellungen umsehen und eigene Theorien oder begrenzte Sets von Theorien entwickeln (,,axes of cohesion“ im Sinne Abbotts). Disziplinen sind also, wie man sieht, historische Einheiten, die sich mit der Autonomisierung des Wissenschaftssystems bilden und die im Zuge dieses Differenzierungsprozesses ihre Einheit ebenfalls autonom konstituieren.

\section{(b) Philosophie als einheitsstiftende Disziplin}

Die Philosophie übernimmt in dieser Situation als neu entstehende Disziplin nun die Aufgabe, die äußerlich gewordene Ordnung der Wissenschaft durch eine intern erzeugte, nur wissenschaftlichen Gesetzen gehorchende Einheit der Wissenschaft zu ersetzen. An die Stelle der alten äußeren Ordnung tritt, wie Kant es formuliert, die „Gesetzgebung der Vernunft“ als umfassendes Prinzip aller Wissenschaft. Dieser Geist prägt die neue wissenschaftliche Universität, beispielsweise die Berliner Universität Wilhelm von Humboldts, welche die alte Staatsdienerschule ablöst. In der genannten ,horizontalen“ Form der Differenzierung fällt es der Philosophie allerdings schwer, die Einheit des Ganzen zu sichern. Die Vorstellung, der Welt wohne eine eigene Ordnung inne, an der Wissenschaft sich orientieren könne, war mit der Ausdifferenzierung der Erfahrungswissenschaften verblasst. Die Vorstellung andererseits, dass die Philosophie eine solche Ordnung von innen heraus schaffen könne, wird ebenfalls durch die Ausdifferenzierung des Wissenschaftssystems konterkariert, innerhalb dessen die Philosophie ja nur noch eine von vielen möglichen Beobachtungsweisen - eben nur eine Disziplin unter vielen - darstellt. Als solcher fällt es ihr schwer, eine privilegierte Kompetenz für die Erzeugung, Erhaltung und Darstellung der Einheit des Wissenschaftssystems für sich zu reklamieren. Sie nimmt zwar als spezialisierte Wissenschaftsphilosophie (und innerhalb dieser als Erkenntnistheorie) die Rolle als Beobachter ein, muss diese Position aber im Laufe der kommenden Jahrhunderte immer wieder gegen Soziologie, Psychologie und heute die neuen Kognitionswissenschaften verteidigen. Und allein aus diesem Konkurrenzverhältnis (und nicht aus dem empirischen Ergebnis der wissenschaftstheoretischen Revierkämpfe) ergibt sich eine kategoriale Schwächung des philosophischen Interpretationsmonopols im Hinblick auf die Einheit der Wissenschaft.

Was deshalb in der Folge als einheitsstiftendes Merkmal in semantischer und sozialstruktureller Hinsicht bleibt, ist eine auf die Wissenschaft als Funktionssystem bezogene Semantik der Wissenschaftlichkeit, die jenseits spezifischer Disziplinbezüge liegen muss. Wissenschaftlichkeit in diesem Sinne bezeichnet für das Gesamtsystem die Regulierung der Anschlussfähigkeit von Kommunikationen über deren Orientierung an Wahrheitsfähigkeit. Vor diesem Hintergrund wird dann die herausragende Rolle der Disziplinen sichtbar. Jede Disziplin reproduziert nämlich je für sich in der Partikularität ihrer Problemstellungen die Unterscheidung von Wissenschaft und Nicht-Wissenschaft an Hand dieses Kriteriums der Wahrheitsfähigkeit (und dann auch allgemeiner: Wissenschaftlichkeit). Mit der Entstehung einer Vielzahl von Disziplinen kann diese Unterscheidung nun nicht mehr in toto vom Funktionssystem erzeugt werden, das jenseits des allgemeinen Kriteriums der Wahrheitsfähigkeit keine allgemein anwendbare Möglichkeit anbietet, die Unterscheidung zu programmieren. Damit ist die Unterscheidung Wissenschaft - Nichtwissenschaft nur innerhalb von 
Disziplinen sinnvoll, nicht zwischen verschiedenen Disziplinen - im Gegenteil, Wahrheitsansprüche, die in verschiedenen Disziplinen Geltung haben, lassen sich nicht notwendig ineinander übersetzen, ja mögen sich sogar bisweilen widersprechen. Angesichts dieser disziplinären Binnendifferenzierung kann das Wissenschaftssystem insgesamt die Selbstbeobachtung „wahr - nicht wahr“ gar nicht anders erzeugen. Disziplinen haben damit in ihrer Spezifität eine Bedeutung für die Identitätssicherung des Funktionssystems Wissenschaft. Nur in ihnen lässt sich die Unterscheidung zwischen der Wissenschaft und ihrer Umwelt je konkret abbilden. Die Instrumente zur Erzeugung dieser Unterscheidung sind Theorien und Methoden. ${ }^{2}$ Dieser Rückzug auf wissenschaftliche „Rationalitätsstandards" bietet gewissermaßen die letzte Möglichkeit, wissenschaftliche von nicht-wissenschaftlichen Kommunikationen zu unterscheiden. Deswegen wird der Bezug auf die Semantik der Wissenschaftlichkeit selbst von denjenigen notwendigerweise in Anspruch genommen, die ansonsten die These der neuen Transdisziplinarität verteidigen. ${ }^{3}$

Die evolutionäre Leistung der Disziplinen besteht, zusammenfassend formuliert, also darin, dem Wissenschaftssystem angesichts (1) steigenden Größenwachstums, (2) fehlender externer Ordnungsleistungen und (3) des Bedarfs an einheitsstiftender Innen-Außen-Abgrenzung sozial-strukturell und semantisch differenzierte Einheiten zur Verfügung zu stellen, die sich über Gegenstände (materiales Feld), Gegenstandsaspekte (Problemstellungen) und darauf bezogene Theoriebildung identifizieren lassen und die mit diesen Eigenschaften das Wissenschaftssystem einerseits mit strukturierter Komplexität („requisite variety“ i. S. Ashbys) ausstatten und andererseits die Einheit des Wissenschaftssystems, seine System-Umwelt-Differenz jeweils mit reproduzieren und sichern.

2 Für die Rechtswissenschaft vgl. beispielsweise Bora (2001).

3 Etwa Mittelstraß $(2005,21)$, der darauf beharrt, dass sich mit Transdisziplinarität „nicht die wissenschaftlichen Rationalitätsstandards und mit diesen die Methoden und die Formen der Theoriebildung“ ändern. 


\section{Inter- und Transdisziplinarität: Eine neue Einheit der Wissenschaft?}

In der solchermaßen differenzierten Wissenschaft stellen sich im zwanzigsten Jahrhundert in Gestalt von Inter- und Transdisziplinarität wiederum neue Differenzierungsformen ein bzw. werden als solche postuliert: Es sei notwendig, inter- oder transdisziplinär zu agieren, heißt es stereotyp zumindest in der wachsenden Zahl „probleminduzierter“ Forschungsfelder. Gegenüber solchen, bisweilen etwas überzogen wirkenden Behauptungen des Neuheitswertes von Interdisziplinarität ist es einerseits wichtig, darauf zu verweisen, dass allem Anschein nach Interdisziplinaritätspostulate nahezu so alt sind wie die Disziplinen selbst. Abbott (2001, 131 f.) datiert das Aufkommen von Interdisziplinaritätsforderungen auf den Zeitraum zwischen 1890 und 1910 und behauptet, die Begeisterung für Interdisziplinarität sei gleichursprünglich mit der Entstehung von Disziplinen. Etwas ketzerisch könnte man auch in Anknüpfung an Hermann Lübbe (1987, 29 f.) sagen, eine Idee der Einheit der Wissenschaft jenseits disziplinärer Spezialisierungen habe es doch immer schon gegeben und an der praktischen Kooperation zwischen den Fächern habe es ebenfalls niemals gemangelt. Jenseits solcher nicht ganz unberechtigter Polemik gegenüber den inflationär sich ausbreitenden Inter- und Transdisziplinaritätspostulaten wird man allerdings an der empirisch beobachtbaren Bedeutungszunahme inter- und transdisziplinärer Arbeitsgebiete kaum Zweifel haben können (dazu noch einmal Brint 2005). Vor dem Hintergrund des im vorangegangenen Abschnitt entfalteten Arguments von der konstitutiven Bedeutung der Disziplinen für das Funktionssystem Wissenschaft stellt sich dann allerdings die Frage nach den Gründen für die Ausbreitung dieser neuen Formen sowie nach deren Effekten. ${ }^{4}$

Worauf reagiert also das Phänomen der Interdisziplinarität? Hermann Lübbe gab trotz der skizzierten Polemik in dem erwähnten Beitrag implizit schon einen Teil der Antwort auf die Frage nach den Entstehungsbedingungen von Interdisziplinarität. Rascher als die Menge der Elemente eines Systems, so formulierte er, wächst die Zahl möglicher Verbindungen zwischen ihnen (a.a.O.). Lübbe suchte die Ursache für Interdisziplinarität mit anderen Worten in dem bereits als Ursache der Disziplinenbildung identifizierten Größenwachstum und dabei insbesondere in wachsender interner Komplexität des Wissenschaftssystems. Interdisziplinarität ist, wenn man dieses Argument ernst nimmt, weiter gehende Strukturbildung, nämlich die Ausbildung von Selektionsbeschränkungen angesichts hoher - genauer: zu hoher - innerer Komplexität. Während die Entwicklung der Disziplinen im vorhin geschilderten historischen Verlauf zunächst noch als Aufbau von ,requisite variety“, von ausreichender innerer Komplexität zum erfolgreichen Operieren in einer komplexen Umwelt zu verstehen ist, findet sie angesichts gesteigerten Wachstums ihre Grenze im Bereich der „adequate complexity“, also dessen, was an innerer Komplexität noch möglich ist, ohne gewissermaßen zur Überhitzung des Systems zu führen. Eine solche, in interne Blockaden mündende Überhitzung droht insbesondere angesichts disziplinärer Überspezialisierung und der wachsenden Schwierigkeit, interne Anschlüsse zu anderen Disziplinen zu erzeugen, also auf der operativen Ebene die Einheit des Wissenschaftssystems zu garantieren.

4 Auch aus einem weiteren Grund kann man die Frage nach der Innovativität von Interdisziplinaritätsforderungen hier unentschieden lassen. Denn für das Argument von der Funktion der Interdisziplinarität ist deren Entstehungszeitraum von zweitrangiger Bedeutung. Entscheidend ist vielmehr die Antwort auf die Frage nach dem Problem, als dessen Lösung sich Inter- und Transdisziplinarität präsentieren können. 
Interdisziplinarität entsteht insofern als erneute Folge des Größenwachstums der Wissenschaft, das an innere Komplexitätsgrenzen heranreicht und dadurch neue Strukturbildung auslöst. Sie ist demnach im Kern so etwas wie ein „Reparaturphänomen“ (Mittelstraß 1987, 152) zur Aufhebung spezialisierungsbedingter Vielfalt und der damit verbundenen Erkenntnisgrenzen, die im System der Wissenschaft letztlich kommunikative Anschlussmöglichkeiten blockieren. ${ }^{5}$

Diese Reparaturleistung kann, wie man bereits an den Disziplinen gesehen hat, nur gelingen, wenn sie die Frage der Einheit der Wissenschaft mit bearbeitet. Ähnlich argumentieren Balsiger (2005), Krüger (1987) oder Heckhausen (1987, 138), der im Anschluss an seine eingangs zitierte Definition von Disziplin Interdisziplinarität im strengen Sinne nur dort erwartet, wo von einem gemeinsamen Problem mit unterschiedlichen Gegenstandsaspekten und unterschiedlichen theoretischen Integrationsniveaus auszugehen ist, also von einem wiederum wissenschaftsintern erzeugten Anpassungsdruck im Sinne der erwähnten ,,adequate complexity“،

Luhmann (1990, 449 f.) nimmt an, dass sich die Wissenschaft überhaupt erst auf der Ebene der Differenzierung nach Forschungsgebieten endgültig von ihrer Umwelt abkopple und sich erst auf dieser Ebene vollständig autonomisiere. Die Disziplinen wären demnach ein notwendiger Zwischenschritt, der allerdings mit der Autonomisierung über Forschungsgebiete nicht verschwindet, sondern innerhalb dieser seine Funktion behält. Interdisziplinarität vollzieht sich, wie Luhmann sagt, ,im Schutz von Disziplinen, die garantieren, dass das, was hier geschieht, als Wissenschaft anerkannt wird“ (ebd., 450). Diese, auch von anderen Autoren geteilte Auffassung weist bereits darauf hin, dass Interdisziplinarität in einem fundamentalen Sinne auf Disziplinen basiert und von deren bereits beschriebenen Leistungen abhängig ist (vgl. auch Klein 1986, 86).

Allerdings ist diese Disziplinbasierung in der Semantik der Interdisziplinarität selbst allenfalls angedeutet, jedoch noch nicht in eindeutiger Weise enthalten. In der wissenschaftlichen Praxis ist der Topos der Interdisziplinarität weniger geeignet, einen klar abgegrenzten Phänomenbereich zu bezeichnen, als vielmehr die Funktion einer Problemerzeugungs- oder jedenfalls Problembezeichnungsformel zu bedienen, indem er auf das durch Größenwachstum, Spezialisierung und Verlust einheitswissenschaftlicher Perspektiven erzeugte Problem hinweist, dessen konkrete Lösung jedoch nicht enthält. Insofern ist Interdisziplinarität in der Tat ein ,übernutzter“ Begriff (Mittelstraß 1987, 156), eine Sammelbezeichnung für allerlei Formen innerwissenschaftlicher Kooperation (Jantsch 1972), die als solche weder über den Anlass solcher Kooperationen, noch über ihre konkrete Form, oder gar über die ihnen innewohnenden Erfolgschancen und Probleme Auskunft gibt.

Angesichts dieser Gemengelage aus relativ schwach ausgeprägten Begrifflichkeiten einerseits und einer weiten empirischen Verbreitung von Inter- und Transdisziplinaritätspostulaten und -semantiken andererseits erscheint es angebracht, den Begriff der Interdisziplinarität und seine möglichen empirischen Erscheinungsformen noch einmal kurz zu reflektieren. Damit soll nicht der Wert von Laborstudien und workplace studies bestritten werden, die zeigen, wie auf der strukturellen Ebene mit den Herausforderungen von Interdisziplinarität im Forschungsalltag umgegangen wird. Das sind schon je konkrete Antworten auf das mit der Interdisziplinaritätssemantik formulierte Problem, um dessen formale Analyse es hier zunächst geht.

5 Man kann diese Diagnose teilen, auch wenn man Abbotts (2001) oben erwähnte empirische Beobachtung der Gleichursprünglichkeit der Disziplinen und der Interdisziplinarität für zutreffend hält. Abbott wendet sich in relativ scharfer Form gegen die Semantik der Interdisziplinarität, ohne die Frage zu beantworten, weshalb diese Semantik Erfolg haben kann. Die Antwort auf diese Frage wird hier in sozialstrukturellen Eigenschaften der disziplinär organisierten Wissenschaft gesucht, während Abbott, der mit einem Modell der „Selbstähnlichkeit“ operiert, die Entstehung von Interdisziplinaritätssemantiken nicht zu erklären braucht. 
Interdisziplinarität weist auf eine spezifische Orientierung des wissenschaftlichen Handelns bzw. Kommunizierens hin. Sie bezeichnet eine Eigenschaft wissenschaftlicher Operationen (Weinrich 1993, 977: mit dem Lehn-Suffix -ität gebildetes Nomen), welche die Disziplinen begrifflich notwendig voraussetzt. Damit wird eine Differenz beobachtet - nämlich die Differenz zwischen Disziplinen - und zwar aus der Perspektive der Einheit dieser Differenz. Es geht also nicht nur um die Unterscheidung zwischen zwei oder mehr Disziplinen, sondern darum, diese Unterscheidung auf einer im Begriff selbst allerdings offen gelassenen Ebene abzubilden, welche die Einheit dieser Differenz bezeichnet. Die Differenz zwischen den Disziplinen geht, das insinuiert der Begriff, in der Einheit von etwas Anderem auf.

Was ist dieses Andere? Wie kann es kommunikativ konstruiert werden? An welche Fälle könnte man denken? Und wie wahrscheinlich ist das Gelingen einer an diesem Anderen der Differenz sich orientierenden wissenschaftlichen Kommunikation? Mit dem Begriff Interdisziplinarität sind alle diese Fragen nicht beantwortet, sondern überhaupt erst aufgeworfen. Das dürfte im Übrigen auch einer der Gründe für die anhaltenden Debatten über Interdisziplinarität sein.

Man kann versuchen, diese doch sehr weitreichenden Fragen sehr einfach in Gestalt von ein paar Gedankenspielen anzugehen, um zu erläutern, welche Konstellationen für die Kommunikation der Einheit einer Differenz es generell gibt. Anders als Röbbeckes „Interdisziplinaritätsstile“ (Röbbecke 2005) orientieren sich diese Konstellationen nicht an einer Kombination aus genau zwei sozialstrukturellen und kulturellen Eigenschaften (Organisationsgrad und kognitive Kopplung), sondern benennen ganz unterschiedliche Reaktionen auf das Dilemma, das mit der Kommunikation der Einheit einer Differenz verbunden ist. Ich skizziere dieses Dilemma und nenne dann einige Konstellationen, welche die wichtigsten Fälle enthalten und die zum Teil auch in der Literatur schon beschrieben sind, ohne dass ich Vollständigkeit für diese Liste reklamieren will.

Das Dilemma der Kommunikation der Einheit einer Differenz besteht in der dabei zu Tage tretenden Paradoxie. Bei der Beobachtung der Einheit einer Differenz können Paradoxien vor allem dann auftauchen, wenn eine Seite der Differenz zur Beschreibung der Einheit benutzt wird. Man könnte das als eine Form des Re-Entry bezeichnen. Bekannteste Beispiele eines solchen Re-entry sind der lügende Kreter bzw. das Paradox des Eubulides: „Dieser Satz ist falsch.“ Solche in Gestalt von auf sich selbst angewendeten Unterscheidungen auftretende Paradoxien (Antinomien oder performative Widersprüche) bewirken Kommunikationsblockaden. Es gibt keine oder jedenfalls keine unaufwendigen Anschlussmöglichkeiten mehr. Kommunikationssysteme entwickeln daher unter solchen Umständen Mechanismen, die es ihnen erlauben, die mögliche Paradoxie unsichtbar zu halten und die Kommunikation gewissermaßen an der Paradoxie vorbei weiter zu führen. Sechs derartige Mechanismen kann man im Hinblick auf die Einheit der Differenz von Disziplinen identifizieren:

\section{Kommunikationsabbruch}

Eine naheliegende Variante besteht darin, die laufende Kommunikation bei Erreichen paradoxaler Kommunikationsblockaden einfach abzubrechen. Der Fall kann mit Blick auf interdisziplinäre Beziehungen beispielsweise vorliegen, wenn etwa nach der Einheit der Unterscheidung von Soziologie und Biologie gefragt und mit soziologischen oder biologischen Konzepten geantwortet wird. Das Beispiel zeigt, dass Kommunikationsabbrüche selbst nur unter spezifischen Rahmenbedingungen eine akzeptable Option darstellen. Vor allem sind dazu nur sehr ephemere Kontakte zwischen den Disziplinen eine Bedingung, die es schadlos erlaubt, auf Kontakte zu verzichten.

\section{Temporalisierung}

Differenzen werden dabei auf der Zeitdimension abgebildet und dadurch aus einer Einheitsperspektive beschreibbar. Im Beobachten des „Davor“ und „Danach“ können sich dann beispielsweise Konzepte des Lernens, der Evolution, des sozialen Wandels und ähnliche herausbilden. Für ein umfassendes Konzept einer Interdisziplinarität, die ihre Einheit zumindest auch in der Sachdimension sucht, dürfte Temporalisierung allerdings kein besonders geeignetes Muster darstellen. 


\section{Ebenenwechsel}

Paradoxien werden auch mit Ebenenwechseln bearbeitet, wie man aus der formalen Logik weiß. In unserem Fall könnte man etwa an die aus der Organisationsforschung (Brunsson) stammende Unterscheidung von talk und action denken. Die Aktivitätsmuster innerhalb von Universitäten beispielsweise bleiben weithin disziplinär, werden aber auf einer Ebene des talk von Interdisziplinaritätssemantiken begleitet, die dort als Metaphern hervorragend einsetzbar sind. Das dürfte sogar ein relativ verbreitetes Muster sein, wie Weingart (1997) angedeutet hat, der Interdisziplinarität in diesem Sinne als paradoxen Diskurs bezeichnet. Das Problem der talk-action-Form besteht in der erforderlichen Latenzsicherung, deren Notwendigkeit aus der Unmöglichkeit oder doch Unziemlichkeit resultiert, die Unterscheidung von talk und action offen zu thematisieren. Mindestens in der jeweiligen disziplinären Reflexionstheorie (also in der Wissenschaftstheorie, -philosophie oder -soziologie) ist das kaum zu leisten. Und Latenzsicherungsmechanismen sind ihrerseits aufwendig (Bora 2002).

\section{Kontingenz bzw. Unentscheidbarkeit}

Grundsätzlich kann man Differenzen auch in der Schwebe lassen. Wer in interdisziplinären Kontexten arbeitet, ist aus der alltäglichen Praxis damit vertraut, dass man dort häufig das Wechseln zwischen Perspektiven, aber auch das gleichzeitige Geltenlassen an und für sich inkompatibler Sichtweisen einsetzt, um disziplinäre Differenzen kommunikativ handhabbar zu machen. Man bezeichnet diese Form der Kooperation dann gerne als Multidisziplinarität. Das Problem dieser Interdisziplinaritätsform besteht darin, dass sie wenig leistungsfähig, weil ohne eigene Beobachtungsschemata mit Strukturaufbauwert ist.

\section{Sachliche Hierarchie}

Eine naheliegende Form des Umgangs mit Paradoxien besteht darin, sie in Hierarchien aufzulösen. Damit ist im Falle der Einheit der Differenz von Disziplinen jede Form ,supradisziplinärer Kontrolle" gemeint, etwa der Versuch der Philosophie, die Einheit der Disziplinen aus der Perspektive einer Einzeldisziplin zu sichern. Husserls „Krisis der europäischen Wissenschaften“ (1977) lässt sich beispielsweise als anspruchsvoller Versuch einer solchen Hierarchisierung von Disziplinen bzw. Wissenschaftskulturen lesen. Auf der organisatorischen Ebene repräsentierten einst die Akademien dieses Modell (Stichweh 1984, 58), die durch die Bindung der neu entstehenden Disziplinen an die Universitäten aber umgekehrt im Laufe des neunzehnten Jahrhunderts zu Institutionen geworden sind, die bewusst interdisziplinäre Kooperation pflegen (ebd., 85). Supradisziplinäre Kontrolle dürfte in Folge des Verlustes einer vorgegebenen Sachordnung kein heute noch gangbarer Weg sein. Denkbar bleiben stattdessen allerdings instrumentelle Beziehungen, die zu asymmetrischen Relationen bzw. Autoritätsgefällen zwischen Disziplinen führen, von denen dann die einen zu Hilfswissenschaften der anderen werden. Man kann das unter der Bezeichnung „Rechtstatsachenforschung“ etwa im Verhältnis zwischen der Jurisprudenz und sozialwissenschaftlichen Fächern beobachten. Das Beispiel zeigt gleichzeitig, dass sich derartige Asymmetrien nur schwer auf Dauer etablieren lassen (Bora et al. 2000), auch wenn sie gerade zur Bearbeitung konkreter Forschungsfelder durchaus hilfreich sein können.

\section{Hyperzyklen}

Schließlich lassen sich im Verhältnis zwischen Disziplinen auch komplexere Wechselbeziehungen denken, die zu sensiblen Anpassungsreaktionen im Sinne „koevolutionärer“ Kopplungen führen. Man kann sich zumindest Fälle vorstellen, in welchen Disziplinen streng autonom (mono-disziplinär im Sinne Heckhausens) operieren, sich dabei aber wechselseitig beobachten und dies vor allem jeweils intern mit Reflexionsleistungen darüber verbinden, welche Effekte und Anpassungsleistungen eigene Operationen in den je anderen Disziplinen erzeugen. Diese Art reflexiver „Hyperzyklen“ ist allerdings recht voraussetzungsreich. Die dafür erforderlichen Selbstund Fremdbeobachtungsleistungen können nur im Hinblick auf eine relativ kleine Zahl jeweils 
anderer Disziplinen überhaupt erbracht werden. Ich bin mir nicht sicher, ob sich dieses Muster empirisch finden ließe, will es aber im Hinblick auf Technik- und Naturwissenschaften auch nicht ausschließen.

Diese sechs Varianten haben eines gemeinsam: die Disziplinen operieren als Einheiten autonom weiter, gehen nicht in einer neuen Einheit auf und benutzen unterschiedliche Mechanismen der Kooperation. Alle Konstellationen sind deshalb in einem strikten Sinne disziplinbasiert. Deshalb ist abschließend eine siebte Konstellation zu erwähnen, in welcher sich aus der paradoxalen Situation eine neue - allerdings disziplinförmige - Emergenzebene bildet.

\section{Emergenz}

Emergenz meint das Entstehen von etwas Neuem, das im Vorliegenden als solches nicht angelegt ist. Er steht als Kürzel für eine Reihe von Fragestellungen, die hier nicht weiter diskutiert werden sollen (vgl. Bora 2003, Hoyningen-Huene 1994, Krohn/Küppers 1992). Jenseits aller ungeklärten Details enthält der Begriff drei Merkmale, nämlich erstens die Annahme eines materialistischen Monismus (gegen Leib-Seele-Dualismus, Vitalismus und ähnliche Strömungen), der jedoch gleichzeitig nicht-reduktionistisch gebaut ist, sowie zweitens die Unterscheidung zweier Ebenen und schließlich drittens die Anwendung einer Beobachtungssprache, die reduktionistische Prädikationen nicht beinhaltet. Unter diesen Voraussetzungen ist Emergenz dann charakterisiert durch, erstens, prinzipielle Nichtvorhersagbarkeit (bzw. das Ausscheiden von Mikrodetermination) und zweitens, eine mehr oder minder stark formulierte Makrodetermination. Emergent sind dann diejenigen Eigenschaften, Strukturen oder Makrobedingungen auf der zweiten Ebene, deren Auftreten aus Eigenschaften der ersten Ebene nicht vorhergesagt werden kann.

Die Eigenschaften der Emergenz scheinen nun exakt auf diejenigen Phänomene zuzutreffen, die landläufig mit dem Attribut des Transdisziplinären belegt werden. Nichtreduzierbarkeit und Nichtvorhersagbarkeit mit Blick auf die disziplinären Strukturen des Wissenschaftssystems sind sicherlich prominente Eigenschaften der typischen transdisziplinären Felder. Sie sind in ihrer thematischen und organisatorischen Struktur nicht auf vorfindliche Disziplinen reduzierbar. Und ihr Entstehen war in dieser Form aus den disziplinären Kontexten heraus nicht unbedingt vorhersagbar. Mittelstraß (2003) - und ähnlich auch Decker und Grunwald (2001) - bezeichnen mit Transdisziplinarität ja eine Form der Interdisziplinarität, die vor allem durch forschungsrelevante Problemlagen und Perspektiven gekennzeichnet ist, auf Disziplinen aufbaut, in ihnen aber nicht angelegt ist und über sie hinausweist. Als Beispiele werden unter anderem immer wieder Nanotechnologie, Hirnforschung und Technikfolgenabschätzung genannt. Transdisziplinarität hat nach diesem Begriffsverständnis vor allem vier Eigenschaften (Mittelstraß 2003, 10): sie ist integrativ, aber nicht holistisch, bildet also keine neuen, umfassenden Erklärungsmuster; sie hebt disziplinäre Engführungen auf, bildet aber keinen neuen disziplinären Zusammenhang; sie ist Wissenschaft und nicht Transwissenschaft; sie ist forschungsgeleitet, nicht theoriegeleitet. Das heißt, sie basiert ebenfalls ganz wesentlich auf disziplinären Kompetenzen, die aber zur Bestimmung von Transdisziplinarität nicht ausreichen (ebd., 12).

Wenn man also Transdisziplinarität als einen Fall dieser siebten Konstellation „Emergenz“ betrachtet, so stellt sich die Frage, wie dieses Neue konkret beschaffen ist, das aus der Einheit der Differenz von Disziplinen emergiert. Mittelstraß' Begriffsverwendung scheint mir gerade in dieser Hinsicht noch unterbestimmt zu sein und damit eine wesentliche Eigenschaft der Transdisziplinarität - deren Disziplinförmigkeit - zu verfehlen. Denn alle vier von ihm genannten Aspekte der Transdisziplinarität sind mit unklaren Einschränkungen ex negativo versehen. Man fragt sich, woran man denn präzise einen transdisziplinären Fall von einem bloß interdisziplinären unterscheiden würde. Insbesondere fällt darüber hinaus auf, dass alle wesentlichen Eigenschaften von Disziplinen in der Charakterisierung transdisziplinärer Felder wieder auftauchen: Problemfeld, Problemperspektive, Integration - allerdings immer verbunden mit der Versicherung, das sei gera- 
de keine Disziplinarität und vor allem keine disziplinentypische theoretische Integration. Wenn disziplinäre Kompetenzen in dieser Hinsicht nicht ausreichen, stellt sich die Frage: was kommt dann noch hinzu? Im Ergebnis scheint Mittelstraß' Antwort auf die allgemeine Eigenschaft der Wissenschaftlichkeit hinauszulaufen, so jedenfalls der Eindruck aus den neueren Publikationen.

Da also Wissenschaftlichkeit (,,wissenschaftliche Rationalitätsstandards“, Mittelstraß 2005, 21) das einheitsstiftende Moment auch in dieser spezifischen Konstellation disziplinärer Differenz darstellt, ist davon auszugehen, dass zumindest solange sich keine viablen funktionalen Äquivalente für Theorien und Methoden als Produktionsmittel der Wissenschaftlichkeit benennen lassen, transdisziplinäre Felder diese identitätsstiftende Leistung ebenso wie die Disziplinen auf der Ebene von Theorien und Methoden erbringen (z. B. in Gestalt von Handlungstheorie, Joas/Kippenberg 2005). Bereits bei Disziplinen haben wir gesehen, dass dies nicht eine einheitliche „Grand Unified Theory“ sein muß. Es genügt schon ein Set von ,paradigmenförmigen“ Theorien. Über Mittelstraß hinausgehend wird man deshalb in transdisziplinären Feldern Disziplinförmigkeit dergestalt erwarten, dass vor dem Hintergrund eines gemeinsamen materialen Feldes und unterschiedlicher Gegenstandsaspekte sich neue theoretische und methodische Integrationsniveaus jedenfalls abzeichnen.

Ich schlage vor, den Begriff der Transdisziplinarität in diesem Sinne zu gebrauchen. Das Neue, das hier im Unterschied zu den zuvor genannten Konstellationen entsteht, hat zwei Komponenten, nämlich zum einen eine disziplinförmige Orientierung an Grundsätzen der Wissenschaftlichkeit, also an Theorien und Methoden, und zum anderen eine gegenüber den etablierten Disziplinen neuartige Themen- und Personalkombination, also ein neues ,settlement “ im Sinne Abbotts. Dieses kann sich in jeder der oben erwähnten Hinsichten (Organisationen, Themen, Fragen, Theorien, vgl. oben S. 5) von alten settlements unterscheiden. Wir könnten dann mit Recht von einem neuen Emergenzniveau sprechen, auf dem Interdisziplinarität eine über die beschriebenen Formen der Kooperation autonomer Disziplinen hinausweisende Gestalt annimmt, welche die Verwendung des Begriffs Transdisziplinarität rechtfertigt.

Mit diesem Verständnis von Transdisziplinarität sind alle Phänomene umfasst, die oben als Beispiele für transdisziplinäre Felder erwähnt wurden. So lassen sich damit auch neuere Entwicklungen in der TA in Gestalt der unlängst angestoßenen Theoriedebatte (vgl. Technikfolgenabschätzung Theorie und Praxis Heft 2007/1) empirisch beobachten. Was man allerdings aus dieser Perspektive für das Verständnis der Transdisziplinarität nicht notwendig braucht, ist der Aspekt der Orientierung an inneren oder äußeren ,Problemen“. Dieser erscheint nicht mehr als das zentrale - womöglich nicht einmal mehr als ein notwendiges - Merkmal transdisziplinärer Organisationsformen. Denn zum einen gibt es starke empirische Hinweise darauf, dass die erwähnten neuen transdisziplinären Zentren an zahlreichen amerikanischen Universitäten viel weniger der organisatorische Ausdruck einer neuen Problemorientierung als vielmehr der Effekt einer neuen ökonomischen Steuerung der Universitäten ist. Monetärer Druck und hochschulpolitische Strategien scheinen diesen Trend besser erklären zu können als zuvor ungelöste theoretische oder praktische Problemstellungen, wie Steven Brint (2005) in einer umfassenden empirischen Studie gezeigt hat. Zum anderen liegt der Problemorientierungsthese eine gewisse Ontologisierung des Problembegriffs zu Grunde. Probleme sind soziale Konstrukte, die vor einem gegebenen sozialstrukturellen und semantischen Hintergrund auftreten. Wer unter welchen Umständen was als Problem definiert und diese Definition gegenüber anderen Akteuren durchhalten kann, ist eine empirische Frage, die wissenssoziologischer Rekonstruktion zugänglich ist und deren Beantwortung gegebenenfalls ein klareres Licht auf die Probleme werfen kann, die zum ,settlement“ transdisziplinärer Felder gehören. Vor diesem Hintergrund ist Problemorientierung allenfalls eine kontingente Eigenschaft transdisziplinärer Felder, die in ihrem ,settlement“" vor allem neue Themen und Organisationsformen aufweisen.

Interdisziplinarität - so kann man nun diesen Abschnitt zusammenfassen - entsteht also aus dem Größenwachstum disziplinär differenzierter Wissenschaft und steht wie diese vor der Aufgabe, die Einheit der Wissenschaft in ihrer Differenziertheit abbilden zu müssen. Dafür gibt es im Wesentli- 
chen zwei Wege: zum einen die - in vielfacher Form denkbare - Kooperation autonomer Disziplinen, d. h. also letztlich den Erhalt disziplinärer Differenzierung und zum anderen die Emergenz neuer Differenzierungsformen, die hier unter dem Stichwort Transdisziplinarität beschrieben wurde. Beide Wege werden letztlich nur ermöglicht durch Prinzipien der Wissenschaftlichkeit, die im Falle der interdisziplinären Kooperation auf Disziplinarität und im Falle der Transdisziplinarität auf Disziplinförmigkeit beruhen.

\section{Die wissenschaftlichen Grundlagen von Transwissenschaft}

Transdisziplinarität, darauf wurde eingangs hingewiesen, wird häufig in Zusammenhang mit außerwissenschaftlichen Problemlagen und Anforderungen gebracht (dazu auch Maasen/Lieven 2006). Im vorhergehenden Abschnitt war demgegenüber auf die disziplinförmige, an der Identität wissenschaftlicher Kommunikationen orientierte Gestalt auch der transdisziplinären Felder hingewiesen worden. Soweit man dieser Lesart folgt, stellt sich dann die Frage nach der Bedeutung der Phänomene, die ganz offenkundig die Grenzen der Wissenschaft transzendieren, sich also als transwissenschaftliche konstituieren. Was folgt aus dem bislang Gesagten für die Möglichkeit eines solchen transwissenschaftlichen Operierens? Ist dieses durch das Argument von der Disziplinförmigkeit a priori ausgeschlossen? Wenn man dies annimmt, wie fügen sich dann transwissenschaftliche Phänomene in den hier entwickelten begrifflichen Rahmen?

Mit diesen Fragen vollziehen wir einen Referenzwechsel. Im Unterschied zu den Disziplinen, die Binnendifferenzierungen des Funktionssystems Wissenschaft darstellen, liegen die im folgenden verhandelten Phänomene der Transwissenschaft auf der Ebene von Organisationen. Organisationen verfügen im Unterschied zu Funktionssystemen über die Fähigkeit, verschiedene Wissensformen gleichzeitig zu prozessieren. Nur deshalb können wir überhaupt über den Einsatz wissenschaftlichen Wissens in nichtwissenschaftlichen Feldern diskutieren. Das ist ein Aspekt, der in den vieldiskutierten Zeitdiagnosen des „mode 2“ und verwandter Argumentationen typischerweise ausgeblendet wird. Das „Ausgreifen“ der Wissenschaft in andere Felder geschieht, wie man leicht zeigen könnte, keineswegs durch einen Wandel wissenschaftlicher Kommunikation bzw. von deren Rationalitätsstandards, sondern besteht in der Verschiebung von Prioritäten in Organisationen (Bora 2005). Dieses Argument soll hier nicht noch einmal en detail entwickelt werden. Vielmehr interessieren im Zusammenhang mit Interdisziplinarität die Struktureigenschaften solchen außerwissenschaftlichen Handelns und dabei insbesondere die Frage, inwiefern dieses auf wissenschaftliche und damit auf disziplinär verfasste Kontexte angewiesen bleibt.

Die Frage nach der außerwissenschaftlichen Einsetzbarkeit von wissenschaftlichem Wissen war im Prinzip, wenngleich in vormoderner Gestalt, bereits den höheren Fakultäten der mittelalterlichen Universität vertraut. Die Erzeugung wissenschaftlich begründeter außerwissenschaftlicher Kompetenz fällt nun im Zuge der funktionalen Differenzierung der Wissenschaft den Professionen zu. Ich kann hier nicht im Einzelnen auf die Grundlagen der Professionssoziologie eingehen. ${ }^{6}$ Hervorzuheben ist jedoch, dass Professionalität sich aus der Perspektive jedenfalls der neueren Professiona-

6 Wissenschaft als Profession ist Thema etwa bei Parsons, Ben-David, Collins und Weingart (vgl. die Nachweise in Münte 2004, 12 ff.). 
lisierungstheorie (Oevermann 1996) nicht in organisatorischen, standesförmigen Strukturen und dem Aufbau professioneller Normsysteme erschöpft. Vielmehr beruht sie auf der Kompetenz eines wissenschaftlich Gebildeten für die Lösung außerwissenschaftlicher Handlungsprobleme. Unter diesen Problemen gibt es einige, die eben den Einsatz wissenschaftlich fundierter Kompetenz voraussetzen, die also mit außerwissenschaftlichen Mitteln nicht alleine zu bewältigen sind. Und dieser Einsatz erfolgt im Fall des Professionellen nicht in technokratisch routinisierbarer Form, sondern verlangt eine komplexe Form der Kooperation, die man als Arbeitsbündnis charakterisieren kann (ausführlicher dazu Bora 2006).

Dieses professionelle Handeln, das im außerwissenschaftlichen Bereich gefragt ist, bleibt konstitutiv angewiesen auf die Sicherung wissenschaftlicher Standards im Inneren, seien sie disziplinärer, inter- oder transdisziplinärer Art. Nur von daher kann, wie wir gesehen haben, die Differenz der Wissenschaft zu ihrer Umwelt gehandhabt werden. Und die steht beim professionellen Agieren in transwissenschaftlichen Feldern stets zur Diskussion. Insofern besteht also - um allfälligen Missverständnissen vorzubeugen - kein Widerspruch zwischen Professionalisierung und disziplinförmiger wissenschaftlicher Kompetenz. Im Gegenteil: jene ist auf diese angewiesen und nur der wissenschaftlich begründeten Profession können wissenschaftliches boundary work (Jasanoff 1987, Gieryn 1983) und die Produktion „sozial robusten Wissens“ (Nowotny 2003) in hybriden Feldern wie etwa der Politikberatung, dem Public Understanding of Science oder eben auch der TA in außerwissenschaftlichen Tätigkeitsfeldern gelingen. Nicht alle außerwissenschaftlichen Aktivitäten, die sich in Organisationen wie zum Beispiel Universitäten abspielen oder an denen Personal des Wissenschaftssystems beteiligt ist, gehören damit auch zur Sphäre wissenschaftlicher Operationen.

Diese Gemengelage von wissenschaftlichen und nicht-wissenschaftlichen Operationen im außerwissenschaftlichen Handeln soll abschließend an einigen aktuellen Beispielen aus der TA erläutert werden, welche drei für die Debatte relevante Fallgestaltungen abdecken. Dabei wird noch einmal deutlich, dass, soweit wissenschaftliche Professionalität gefragt ist, diese nur über wissenschaftsinterne, ergo disziplinär verfasste Mechanismen gesichert werden kann.

(1) TA wird - in Gestalt der partizipativen TA - bisweilen als kommunikative Arena betrieben, in welcher sich unterschiedliche gesellschaftliche Felder treffen und über die Bewertung von Technologie mit dem Ziel der Erzeugung von Entscheidungen streiten. Solche Arenen sind aber im Hinblick auf dieses Ziel der Bildung oder Vorbereitung kollektiv bindender Entscheidungen Teil des politischen Systems, auch wenn sie dazu wissenschaftliches Wissen benötigen. Soweit TA-Akteure dort auftreten, betreiben sie eben bereits durch die Beteiligung an solchen Entscheidungs- oder Entscheidungsvorbereitungsprozessen Politik, selbst wenn dabei wissenschaftliches Wissen instrumentell zum Einsatz kommt, was ja ein ganz alltägliches Phänomen ist und keiner terminologischen Überhöhung - wie etwa „Gesellschaftsberatung“ - bedarf. „Die Worte, die man braucht, sind dann nicht Mittel wissenschaftlicher Analyse, sondern politischen Werbens um die Stellungnahme der anderen. Sie sind nicht Pflugscharen zur Lockerung des Erdreiches des kontemplativen Denkens, sondern Schwerter gegen die Gegner: Kampfmittel.“ (Weber 1917/19, 1964, 325). Solche Arenen sind sehr wichtige Instrumente im politischen Kontext, aber man sollte sich darüber im Klaren sein, dass sie insofern nicht Teil von Wissenschaft sind und es auch nicht durch die Beteiligung von Personen werden, die sonst an Labortischen und hinter Kathedern stehen. Wenn und soweit also nichtwissenschaftliche Relevanzen im Vordergrund stehen, besteht in dem oben genannten Sinne insofern kein Professionalisierungsbedarf.

(2) Etwas anders verhält es sich dort, wo es per definitionem um wissenschaftliche Politikberatung geht. Sie kann als Beispiel für das Problem der Anschlussfähigkeit wissenschaftlicher Kommunikationen außerhalb der Wissenschaft gelten, bei der nun in der Tat Professionalisierungsbedarf anzunehmen ist. Oft genügt dazu schon, dass die eben erwähnten Arenen der pTA in eine eindeutige Beziehung zu politischen Entscheidungsinstanzen gesetzt werden. Bei TA als Politikberatung handelt es sich um eine ins Außerwissenschaftliche reichende Aktivität, mit der beispielsweise inte- 
grative Regulierungsansätze gesucht werden, die kognitive, normative und evaluative Dimensionen und die betreffenden Funktionssysteme integrieren. Hierzu braucht es, wie ich andernorts zu begründen versucht habe, multireferentielle Organisationen und Verfahren (Bora 2006). Und es braucht als wissenschaftsbasierte Komponente dieses außerwissenschaftlichen Feldes Professionalisierung, die allerdings Orientierungswissen immer auf wissenschaftlicher Basis in dem eben diskutierten Sinne zur Verfügung stellt.

Die Grenzen zwischen TA als Teil der Politik und als Instrument der Politikberatung sind in der Empirie oft fließend. Das scheint mir unter anderem ein Grund für beobachtbare Akzeptanzprobleme dieser Verfahren zu sein. Klare Funktionsbestimmungen und darauf abgestellte Wahl von Verfahrensformen sind deshalb unabdingbar (Abels/Bora 2004).

(3) Schließlich ist auch die Einbeziehung der Laien in die Produktion wissenschaftlichen Wissens zu erwähnen. Immer wieder wird, oft im Zusammenhang mit Problemorientierung, die Einbeziehung außerwissenschaftlichen Wissens in die Wissenschaft - und natürlich insbesondere in die TA - thematisiert. Dazu scheint mir zunächst wichtig zu sagen, dass selbstverständlich jede Erfahrungswissenschaft andauernd außerwissenschaftliche Daten beobachtet und sammelt. Sie tut dies mit sehr unterschiedlichen Mitteln. Unter anderem bezieht sie auch nichtwissenschaftliche Akteure ein. Traditionelle Beispiele sind die Botanik oder der Naturschutz, wo dies jedenfalls zeitweilig eine sehr wichtige Rolle gespielt hat. Dieser Sachverhalt erscheint jedoch im Zusammenhang mit der Interdisziplinaritäts- und Wissenschaftlichkeits-Thematik als gänzlich unproblematisch. Einen Professionalisierungsbedarf gibt es hier mangels eines außerwissenschaftlichen Handlungsproblems nicht. Die unter Beteiligung von Laien zu beantwortenden Fragen sind rein innerwissenschaftlicher Natur. Die von Laien besorgten Daten und Erkenntnisse werden nach den Grundsätzen wissenschaftlicher Methodik ausgewertet.

Auch im Falle der Normwissenschaften und des sogenannten Orientierungswissens verhält es sich nicht anders. Denn auch die von Laien mobilisierten normativen Gesichtspunkte und Präferenzen sind letztlich in einer gemäß den Theorien und Methoden - etwa der Ethik oder des Rechts - eingerichteten und insofern wissenschaftlichen Argumentation zu prüfen, wenn und soweit sie in den Operationen innerhalb des Wissenschaftssystems anschlussfähig bleiben sollen.

Wo dies aber gar nicht das Ziel ist - sondern beispielsweise die „Demokratisierung der Expertise“ (Nowotny 2003) im Vordergrund steht -, handelt es sich, wie im eben erwähnten Fall schon gesagt, um etwas anderes als Wissenschaft. Es stellen sich insofern keine Fragen der Wissenschaftlichkeit.

Kurz und gut: TA trägt vielfach transwissenschaftliche Züge, das würde niemand bestreiten wollen. Die Beispiele sollten plausibel machen, dass in diesem Bereich mindestens drei verschiedene Fälle denkbar sind. Soweit wissenschaftliche Anschlussfähigkeit überhaupt eine Rolle spielt, kommen in allen diesen Fällen wiederum die bereits ausführlich erörterten Mechanismen der Sicherung von Wissenschaftlichkeit zum Tragen. 


\section{Zusammenfassung: Disziplinbasierung und Disziplinförmigkeit}

Inter- und Transdisziplinarität, darin sind sich alle relevanten Autoren einig, sind keine vom Gefüge wissenschaftlicher Disziplinen losgelösten Phänomene. In diesem Beitrag wurde darüber hinaus die These vertreten, dass beide, Inter- wie Transdisziplinarität, in ihrer Struktur den Disziplinen nahe stehen, da sie entweder aus disziplinären Formen gebildet werden (Disziplinbasierung) oder selbst, gleichsam auf neuem Emergenzniveau disziplinäre Form annehmen (Disziplinförmigkeit). Für den Fall neuer, bestehende Disziplinen thematisch, organisatorisch und personell überschreitender disziplinförmiger Felder wurde vorgeschlagen, den Begriff der Transdisziplinarität zu reservieren, der sich ansonsten nur sehr unscharf von demjenigen der Interdisziplinarität abhebt.

Die wesentlichen Eigenschaften der so bestimmten Inter- und Transdisziplinarität können dann auf neu im Entstehen begriffene Forschungsfelder angewendet werden. TA ist ein solches hybrides Feld. Man kann an diesem Beispiel gerade im Hinblick auf transwissenschaftliche Felder auf eine gewisse Gefahr der aktuellen wissenschaftssoziologischen Debatte aufmerksam machen, die darin liegt, dass aus etwas unklaren Begriffen und historisch wenig tiefenscharfen empirischen Beobachtungen weitreichende theoretische Schlussfolgerungen gezogen werden. Diese Gefahr der begrifflich-theoretischen Übergeneralisierung kann durch eine differenzierende Begriffwahl vermieden werden.

Inhaltlich ist auf dieser Basis der derzeitige Stand des Unternehmens TA im Inneren als heterogen mit unklarer Tendenz zu charakterisieren. Technology Assessment trägt offenkundig sowohl die Züge einer Kooperation autonomer Disziplinen, als auch die einer sich bildenden Transdisziplin. Beide basieren, wie darzulegen versucht wurde, mindestens auf disziplinförmigen Strukturen.

Dasselbe gilt letztlich auch für die im transwissenschaftlichen Bereich mögliche Professionalisierung nach außen. Sie erfordert, das wurde mit den letzten Beispielen kurz angerissen, ebenfalls Wissenschaftlichkeit, also Disziplinbasierung oder Disziplinförmigkeit autonomer Wissenschaft im Inneren.

Alles in allem findet damit TA als Transdisziplin und als professionalisiertes Feld ,im Schutz der Disziplinen“, also disziplinenbasiert oder disziplinförmig statt. Worin dieser Schutz der Disziplinen besteht und weshalb er für ein wissenschaftlich ausgerichtetes Vorhaben erforderlich ist, wurde in diesem Beitrag darzulegen versucht. 


\section{Literatur}

Abels, G., Bora, A., 2004, Demokratische Technikbewertung, Bielefeld: transcript.

Abbott, A., 2001, Chaos of Disciplines, Chicago/London: Chicago University Press.

Ashby, W. R., 1956, An Introduction to Cybernetics, London: Chapman \& Hall.

Balsiger, P. W., 2005, Transdisziplinarität, München: Fink.

Bora, A., 1999, Differenzierung und Inklusion. Partizipative Öffentlichkeit im Rechtssystem moderner Gesellschaften, Baden-Baden: Nomos.

Bora, A., 2000/2005, Zum soziologischen Begriff des Diskurses, Manuskript, Bielefeld 2000 und 2005, 28 S., http://www.uni-bielefeld.de/iwt/personen/bora/pdf/Alfons \%20Bora \%20Ms \%20Diskurs \%202000 \%20and \%202005.pdf (zuletzt besucht 7.11.2007).

Bora, A., 2001, Referenz und Resonanz - Zur Funktion von Methoden in Rechtstheorie, Rechtslehre und Rechtspoiesis, Rechtstheorie 32(2/3), 259-272.

Bora, A., 2002, Ökologie der Kontrolle, Technikregulierung unter der Bedingung von Nicht-Wissen, in: Engel, Ch., Halfmann, J., Schulte, M., (Hg.), Wissen, Nichtwissen, unsicheres Wissen, Baden-Baden: Nomos, 253-275.

Bora, A., 2003, „Whatever its causes“ - Emergenz, Koevolution und strukturelle Kopplung, in: Wenzel, U., Holz, K., Bretzinger, B., (Hg.), Subjekte und Gesellschaft. Zur Konstitution von Sozialität, Weilerswist: Velbrück, 117-138.

Bora, A., 2005, Rezension: Helga Nowotny, Peter Scott und Michael Gibbons: Wissenschaft neu denken. Wissenschaft und Öffentlichkeit in einem Zeitalter der Ungewissheit, Weilerswist: Velbrück 2004, Kölner Zeitschrift für Soziologie und Sozialpsychologie 57(4), 755-757.

Bora, A., 2006, Technology Assessment als Politikberatung, in: Weingart, P., Taubert, N. C., (Hg.), Das Wissensministerium. Ein halbes Jahrhundert Forschungs- und Bildungspolitik in Deutschland, Weilerswist: Velbrück, 92-114.

Bora, A., Höland, A., Jansen, D., Lucke, D., Ludwig-Mayerhofer, W., Machura, St., Teubner, G., 2000, Rechtssoziologie , auf der Grenze', Editorial, Zeitschrift für Rechtssoziologie 21(2), 319-326.

Brint, S., 2005, Creating the Future: ,New Directions' in American Research Universities, Minerva 43(1), 25-50.

Decker, M., Grunwald, A., 2001, Rational Technology Assessment as Interdisciplinary Research, in: Decker, M., (Hg.), Interdisciplinarity in Technology Assessment. Implemention and its Chances and Limits, Berlin: Springer, 33-60.

Funtowicz, S. O., Ravetz, J. R., 1993, The Emergence of Post-Normal Science, in: v. Schomberg, R., (Hg.), Science, Politics and Morality. Scientific Uncertainty and Decision Making, Dordrecht: Kluwer, 85-123.

Gibbons, M., Limoges, C., Nowotny, H., Schwartzman, S., Scott, P., Trow, M., 1994, The New Production of Knowledge, London: Sage.

Gieryn, T. F., 1983, Boundary-Work and the Demarcation of Science from Non-Science: Strains and Interests in Professional Ideologies of Scientists, American Sociological Review 48(6), 781-795. 
Grunwald, A., Schmidt, J. C., 2005, Method(olog)ische Fragen der Inter- und Transdisziplinarität. Wege zu einer praxisstützenden Interdisziplinaritätsforschung, Technikfolgenabschätzung Theorie und Praxis 14(2), 4-11.

Hausendorf, H., Bora A., (Hg.), 2006, Analysing Citizenship Talk. Social positioning in political and legal decision-making processes, Discourse Approaches to Politics, Society and Culture 19, Amsterdam: John Benjamins.

Heckhausen, H., 1987, „Interdisziplinäre Forschung” zwischen Intra-, Multi- und Chimären-Disziplinarität, in: Kocka, J., (Hg.), Interdisziplinarität, Praxis - Herausforderungen - Ideologie, Frankfurt/M.: Suhrkamp, 129-145.

Hoyningen-Huene, P., 1994, Zu Emergenz, Mikro- und Makrodetermination, in: Lübbe, W., (Hg.), Kausalität und Zurechnung. Über Verantwortung in komplexen kulturellen Prozessen, Berlin/New York: de Gruyter, 165-195.

Husserl, E., 1977, Die Krisis der europäischen Wissenschaften und die transzendentale Phänomenologie: Eine Einleitung in die phänomenologische Philosophie, Hamburg: Meiner.

Jantsch, E., 1972, Towards Interdisciplinarity and Transdisciplinarity in Education and Innovation, in: CERI, (Hg.), Interdisciplinarity. Problems of Teaching and Research in Universities, Paris: OECD, 97-120.

Jasanoff, S., 1987, Contested boundaries in policy-relevant science. Social Studies of Science $17(2), 195-230$.

Joas, H., Kippenberg, H., 2005, Interdisziplinarität als Lernprozeß. Erfahrungen mit einem handlungstheoretischen Forschungsprogramm, Göttingen: Wallstein.

Klein, J., 1986, The Dialectic and Rhetoric of Disciplinarity and Interdisciplinarity, in: Chubin, D., et al., (Hg.), Interdisciplinarity Analysis and Research, Mt. Airy: Lomond, 85-100.

Krohn, W., Küppers, G., (Hg.), 1992, Emergenz: Die Entstehung von Ordnung, Organisation und Bedeutung, Frankfurt/M.: Suhrkamp.

Krüger, L., 1987, Einheit der Welt - Vielheit der Wissenschaft, in: Kocka, J., (Hg.), Interdisziplinarität, Praxis - Herausforderungen - Ideologie, Frankfurt/M.: Suhrkamp, 106-125.

Lübbe, H., 1987, Helmut Schelsky und die Interdisziplinarität. Zur Philosophie gegenwärtiger Wissenschaftskultur, in: Kocka, J., (Hg.), Interdisziplinarität, Praxis - Herausforderungen Ideologie, Frankfurt/M.: Suhrkamp, 17-33.

Luhmann, N., 1990, Die Wissenschaft der Gesellschaft, Frankfurt/M.: Suhrkamp

Maasen, S., Lieven, O., 2006, Transdisciplinarity: a new mode of governing science? Science and Public Policy 33(2996), 399-410.

Mittelstraß, J., 1987, Die Stunde der Interdisziplinarität? in: Kocka, J., (Hg.), Interdisziplinarität, Praxis - Herausforderungen - Ideologie, Frankfurt/M.: Suhrkamp, 152-158.

Mittelstraß, J., 2003, Transdisziplinarität - wissenschaftliche Zukunft und institutionelle Wirklichkeit, Konstanz: UVK.

Mittelstraß, J., 2005, Methodische Interdisziplinarität, Technikfolgenabschätzung - Theorie und Praxis 14(2), 18-24.

Münte, P., 2004, Die Autonomisierung der Erfahrungswissenschaften im Kontext frühneuzeitlicher Herrschaft: fallrekonstruktive Analysen zur Gründung der Royal Society, Frankfurt/M.: Humanities Online. 
Nowotny, H., 2003, Democratising expertise and socially robust knowledge, Science and Public Policy 30(3), 151-157.

Nowotny, H., Scott, P., Gibbons, M., 2001, Re-thinking science: knowledge and the public in an age of uncertainty, Cambridge: Polity Press.

Oevermann, U., 1996, Theoretische Skizze einer revidierten Theorie professionalisierten Handelns, in: Combe, A., Helsper, W., (Hg.), Pädagogische Professionalität, Frankfurt/M.: Suhrkamp, 70-182.

Ravetz, J. R., 1999, What is Post-Normal Science? FUTURES 31(7), 647-653.

Ravetz, J. R., 2004, The post-normal science of precaution, FUTURES 36(3), 347-357.

Röbbecke, M., 2005, Bedingungen von Interdisziplinarität in der Forschung, Technikfolgenabschätzung - Theorie und Praxis 14(2), 39-44.

Stichweh, R., 1984, Zur Entstehung des modernen Systems wissenschaftlicher Disziplinen, Frankfurt/M.: Suhrkamp.

Weber, M., 1917/19, Wissenschaft als Beruf, in: Winckelmann, J., (Hg.), Soziologie, weltgeschichtliche Analysen, Politik, 3. Aufl., Stuttgart: Kröner 1964.

Weingart, P., 1997, Interdisziplinarität - der paradoxe Diskurs, Ethik und Sozialwissenschaften, $8(4), 521-529$.

Weinrich, H., 1993, Textgrammatik der deutschen Sprache, Mannheim: Duden. 


\section{Bisher erschienene manu:scripte}

ITA-0I-0I Gunther Tichy, Walter Peissl (12/2001): Beeinträchtigung der Privatsphäre in der Informationsgesellschaft. <http://www.oeaw.ac.at/ita/pdf/ita_0I_0I.pdf>

ITA-0I-02 Georg Aichholzer(12/2001): Delphi Austria: An Example of Tailoring Foresight to the Needs of a Small Country. <http://www.oeaw.ac.at/ita/pdf/ita_0I_02.pdf>

ITA-0I-03 Helge Torgersen, Jürgen Hampel (12/2001): The Gate-Resonance Model: The Interface of Policy, Media and the Public in Technology Conflicts.

<http://www.oeaw.ac.at/ita/pdf/ita_0l_03.pdf>

ITA-02-0I Georg Aichholzer (01/2002): Das ExpertInnen-Delphi: Methodische Grundlagen und Anwendungsfeld ,Technology Foresight“. <http://www.oeaw.ac.at/ita/pdf/ita_02_0I.pdf>

ITA-02-02 Walter Peissl (01/2002): Surveillance and Security - A Dodgy Relationship. $<$ http://www.oeaw.ac.at/ita/pdf/ita_02_02.pdf $>$

ITA-02-03 Gunther Tichy (02/2002): Informationsgesellschaft und flexiblere Arbeitsmärkte. $<$ http://www.oeaw.ac.at/ita/pdf/ita_02_03.pdf >

ITA-02-04 Andreas Diekmann (06/2002): Diagnose von Fehlerquellen und methodische Qualität in der sozialwissenschaftlichen Forschung. <http://www.oeaw.ac.at/ita/pdf/ita_02_04.pdf>

ITA-02-05 Gunther Tichy (10/2002): Over-optimism Among Experts in Assessment and Foresight. <http://www.oeaw.ac.at/ita/pdf/ita_02_05.pdf>

ITA-02-06 Hilmar Westholm (12/2002): Mit eDemocracy zu deliberativer Politik? Zur Praxis und Anschlussfähigkeit eines neuen Mediums. <http://www.oeaw.ac.at/ita/pdf/ita_02_06.pdf>

ITA-03-0I Jörg Flecker und Sabine Kirschenhofer (01/2003): IT verleiht Flügel? Aktuelle Tendenzen der räumlichen Verlagerung von Arbeit. $<$ http://www.oeaw.ac.at/ita/pdf/ita_03_0l.pdf>

ITA-03-02 Gunther Tichy (11/2003): Die Risikogesellschaft - Ein vernachlässigtes Konzept in der europäischen Stagnationsdiskussion.

$<$ http://www.oeaw.ac.at/ita/pdf/ita_03_02.pdf>

ITA-03-03 Michael Nentwich (11/2003): Neue Kommunikationstechnologien und Wissenschaft - Veränderungspotentiale und Handlungsoptionen auf dem Weg zur Cyber-Wissenschaft. <http://www.oeaw.ac.at/ita/pdf/ita_03_03.pdf>

ITA-04-0I Gerd Schienstock (1/2004): Finnland auf dem Weg zur Wissensökonomie - Von Pfadabhängigkeit zu Pfadentwicklung. <http://www.oeaw.ac.at/ita/pdf/ita_04_0I.pdf>

ITA-04-02 Gunther Tichy (6/2004): Technikfolgen-Abschätzung: Entscheidungshilfe in einer komplexen Welt. <http://www.oeaw.ac.at/ita/pdf/ita_04_02.pdf>

ITA-04-03 Johannes M. Bauer (11/2004): Governing the Networks of the Information Society - Prospects and limits of policy in a complex technical system.

<http://www.oeaw.ac.at/ita/pdf/ita_04_03.pdf>

ITA-04-04 Ronald Leenes (12/2004): Local e-Government in the Netherlands: From Ambitious Policy Goals to Harsh Reality. <http://www.oeaw.ac.at/ita/pdf/ita_04_04.pdf>

ITA-05-0I Andreas Krisch (01/2005): Die Veröffentlichung des Privaten - Mit intelligenten Etiketten vom grundsätzlichen Schutz der Privatsphäre zum Selbstschutz-Prinzip. <http://www.oeaw.ac.at/ita/pdf/ita_05_0I.pdf> 
ITA-05-02 Petra Grabner (12/2005): Ein Subsidiaritätstest - Die Errichtung gentechnikfreier Regionen in Österreich zwischen Anspruch und Wirklichkeit.

<http://www.oeaw.ac.at/ita/pdf/ita_05_02.pdf>

ITA-05-03 Eva Buchinger (12/2005): Innovationspolitik aus systemtheoretischer Sicht - Ein zyklisches Modell der politischen Steuerung technologischer Innovation. $<$ http://www.oeaw.ac.at/ita/pdf/ita_05_03.pdf >

ITA-06-0I Michael Latzer (06/2006): Medien- und Telekommunikationspolitik: Unordnung durch Konvergenz - Ordnung durch Mediamatikpolitik. $<$ http://epub.oeaw.ac.at/ita/ita-manuscript/ita_06_0I.pdf $>$

ITA-06-02 Natascha Just, Michael Latzer, Florian Saurwein (09/2006): Communications Governance: Entscheidungshilfe für die Wahl des Regulierungsarrangements am Beispiel Spam. <http://epub.oeaw.ac.at/ita/ita-manuscript/ita_06_02.pdf >

ITA-06-03 Veronika Gaube, Helmut Haberl (10/2006): Sozial-ökologische Konzepte, Modelle und Indikatoren nachhaltiger Entwicklung: Trends im Ressourcenverbrauch in Österreich. <http://epub.oeaw.ac.at/ita/ita-manuscript/ita_06_03.pdf>

ITA-06-04 Maximilian Fochler, Annina Müller (11/2006): Vom Defizit zum Dialog? Zum Verhältnis von Wissenschaft und Öffentlichkeit in der europäischen und österreichischen Forschungspolitik.

<http://epub.oeaw.ac.at/ita/ita-manuscript/ita_06_04.pdf>

ITA-06-05 Holger Floeting (11/2006): Sicherheitstechnologien und neue urbane Sicherheitsregimes. $<$ http://epub.oeaw.ac.at/ita/ita-manuscript/ita_06_05.pdf>

ITA-06-06 Armin Spök (12/2006): From Farming to „Pharming”- Risks and Policy Challenges of Third Generation GM Crops. <http://epub.oeaw.ac.at/ita/ita-manuscript/ita_06_06.pdf>

ITA-07-0I Volker Stelzer, Christine Rösch, Konrad Raab (3/2007): Ein integratives Konzept zur Messung von Nachhaltigkeit - das Beispiel Energiegewinnung aus Grünland. $<$ http://epub.oeaw.ac.at/ita/ita-manuscript/ita_07_0I.pdf $>$

ITA-07-02 Elisabeth Katzlinger (3/2007): Big Brother beim Lernen: Privatsphäre und Datenschutz in Lernplattformen.

$<$ http://epub.oeaw.ac.at/ita/ita-manuscript/ita_07_02.pdf>

ITA-07-03 Astrid Engel, Martina Erlemann (4/2007): Kartierte Risikokonflikte als Instrument reflexiver Wissenspolitik. <http://epub.oeaw.ac.at/ita/ita-manuscript/ita_07_03.pdf>

ITA-07-04 Peter Parycek (5/2007): Gläserne Bürger - transparenter Staat? Risiken und Reformpotenziale des öffentlichen Sektors in der Wissensgesellschaft. $<$ http://epub.oeaw.ac.at/ita/ita-manuscript/ita_07_04.pdf>

ITA-07-05 Helge Torgersen (7/2007): Sicherheitsansprüche an neue Technologien - das Beispiel Nanotechnologie. <http://epub.oeaw.ac.at/ita/ita-manuscript/ita_07_05.pdf>

ITA-07-06 Karen Kastenhofer (9/2007): Zwischen „schwacher“ und „starker“ Interdisziplinarität. Die Notwendigkeit der Balance epistemischer Kulturen in der Sicherheitsforschung zu neuen Technologien. <http://epub.oeaw.ac.at/ita/ita-manuscript/ita_07_06.pdf>

ITA-07-07 Ralf Lindner, Michael Friedewald (9/2007): Gesellschaftliche Herausforderungen durch, ,intelligente Umgebungen. Dunkle Szenarien als TA-Werkzeug. $<$ http://epub.oeaw.ac.at/ita/ita-manuscript/ita_07_07.pdf>

ITA-07-08 Alfons Bora (11/2007): Die disziplinären Grundlagen der Wissenschaft. $<$ http://epub.oeaw.ac.at/ita/ita-manuscript/ita_07_08.pdf $>$ 